\title{
Perirhinal Cortex Neuronal Activity is Actively Related to Working Memory in the Macaque
}

\author{
Christian Hölscher and Edmund T Rolls \\ Oxford University, Department of Experimental Psychology \\ South Parks Road, Oxford OX1 3UD, UK
}

SUMMARY

Lesion studies suggest that the perirhinal cortex plays a role in object recognition memory. To analyze its role, we recorded the activity of single neurons in the perirhinal cortex in a rhesus monkey (Macaca mulatta) performing a delayed matching-to-sample task with up to four intervening stimuli. Certain neurons (40 of 90 analyzed) showed a smaller response to an image when it was shown the second time within a trial (as a match image) than when it had been shown (as a sample image) the first time. A new finding was that the perirhinal cortex neurons were actively reset between trials: when a particular image was shown as a sample on a succeeding trial, the response was much larger than when it had been shown as a match image a short time previously on the previous trial. This resetting between trials appears to reflect the operation of an active working memory process rather than a passive temporal decay in a neuronal response. The results thus provide evidence that the perirhinal cortex plays an active role in visual working memory, perhaps in association with other brain areas such as the prefrontal cortex.

Reprint requests to: Christian Hölscher, Present address: Tübingen University, Dept Cognitive Neuroscience, Auf der Morgenstelle 28, 72076 Tübingen, Germany

email: christian.hoelscher@uni-tuebingen.de

\section{KEYWORDS}

delayed match-to-sample, single unit recording, electrophysiology, object discrimination, primate, learning

\section{INTRODUCTION}

The perirhinal cortex receives connections from the inferior temporal visual cortex (IT) and also from olfactory and somatosensory cortical areas (Suzuki \& Amaral, 1994; Suzuki \& Amaral, 1994). Lesion studies show that the functions of the perirhinal cortex are different from those of the inferior temporal visual cortex. For example, macaques with lesions of the middle temporal gyrus (part of TE/IT) were severely impaired in a color discrimination task, whereas monkeys with perirhinal lesions were unimpaired on this task. Nevertheless, in a delayed nonmatching-to-sample task with delays and extended list lengths. monkeys with perirhinal cortex lesions were severely impaired both in relearning the basic delayed nonmatching-to-sample task and on the postoperative performance test. In contrast, monkeys with TE lesions were only mildly affected in relearning the basic nonmatching task and were unimpaired on the postoperative performance test (Buckley et al., 1997)

Previous studies in which single neurons were recorded showed that perirhinal cortex neurons 
respond to visual stimuli in a way that does not reflect the identity of the stimulus, but that can for different neurons reflect whether stimuli are novel, are familiar, or have been seen very recently (Brown \& Xiang, 1998; Xiang \& Brown, 1998). The results of other studies have shown that in paired association learning tasks (known to be affected by lesions of the perirhinal and entorhinal cortex), the ability of inferotemporal cortex neurons to reflect associations between picture pairs was lost after disruption of projections from rhinal to the inferotemporal neurons, while the ability of the neurons to respond to an individual visual stimulus was left intact (Miyashita et al., 1996; Miyashita et al., 1998). These studies indicate that the IT and perirhinal cortex work closely together, and that certain complex visual discrimination and recognition processes in IT (Rolls, 2000) require a functional perirhinal cortex.

To analyze further the functions of the perirhinal cortex in memory and object recognition we recorded neurons in the perirhinal cortex during the presentation of images in a delayed match to sample task. To examine the temporal aspects of the short term memory, we used a task design incorporating intervening stimuli between the sample and match so that we could analyze if the neurons had responses reflecting whether the sample had been seen even when other stimuli intervened. The task also allowed us to test whether this was an active memory that was reset by each new sample at the start of a trial, or whether the neurons more passively reflected recently seen stimuli.

\section{EXPERIMENTAL}

We investigated the activity of single neurons in the perirhinal cortex in a rhesus monkey (Macaca mulatta weighing $3 \mathrm{~kg}$ ) performing a delayed matching-to-sample task with up to three intervening stimuli between the sample and match. Single-cell recordings were conducted using single neuron tungsten microelectrodes (tip size less than 10 microns) insulated with a plastic coating except for the tip (FHC, U.S.A) during daily recording sessions. The microelectrodes were stereotaxically guided, and the location of the microelectrodes was reconstructed on each track using $\mathrm{X}$-rays and subsequent histological reconstruction using microlesions made on selected tracks. A recording system filtered and amplified the signal, and spikes were sampled and later sorted and cluster cut offline using the Datawave (U.S.A.) Discovery software. The neurophysiological methods used here are described in detail elsewhere (Booth \& Rolls, 1998).

All procedures, including preparative and subsequent ones, were carried out in accordance with the NIH Guide for the Care and Use of Laboratory Animals and the guidelines of The Society for Neuroscience, and were licensed under the U.K. Animals (Scientific Procedures) Act, 1986. The delayed matching-to-sample task consisted of the presentation of an image of an object on a monitor and the successive presentation of four images afterward. One of the four images was the same as the sample image, and when the monkey licked during this match image, he obtained fruit juice reward. Licks at any other time resulted in saline delivery. Image presentation times were $1.2 \mathrm{~s}$, with a delay between images of $500 \mathrm{~ms}$ (see Fig. 1). Each image was presented between 20 and 50 times a day. In experiment 1 , a set of images was shown for 7 days. In experiment 2, a new set of images was added, and cells were recorded for 13 days. Then, in experiment 3 a further set of novel images was added, and neurons were recorded for a further 7 days. Finally, in experiment 4, more novel images were added and recordings were made for another 9 days. 


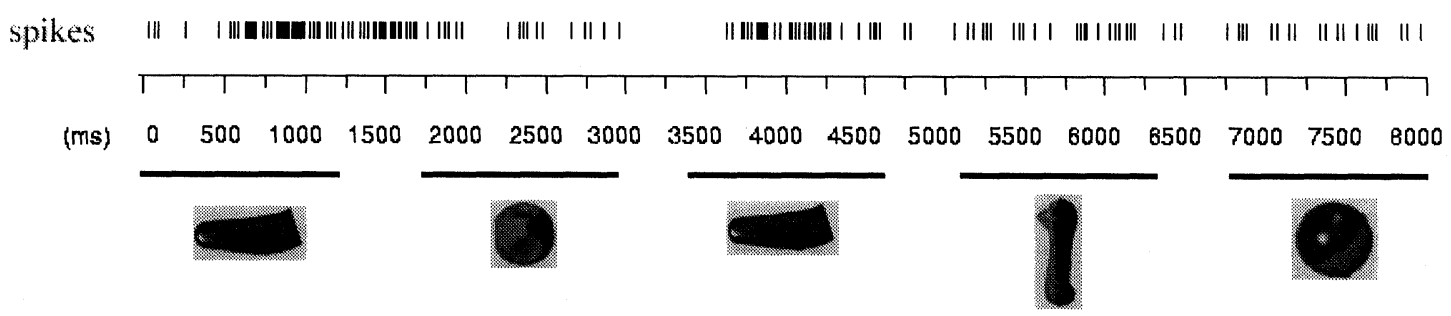

Fig. 1: Experiment 1: Example of the spike trains of one neuron measured during the presentation of images in the delayed match to sample task. Some of the images shown to the monkey are illustrated. The originals were in color but are shown here as 265 grey-level reproductions.

After the experiments were done, 12 marker microlesions were made by passing current $(100 \mu \mathrm{A}$ for $100 \mathrm{~s})$ through microelectrodes at sites that were measured in the X-ray coordinate system being used. The monkey was anesthetized and cardially perfused with saline and formaline. The brain was fixed in buffered formaline (10\%), left in a $30 \%$ sucrose solution over night, and then cut on a cryostat. Sections were stained with cresyl violet stain, the location of the lesions was identified, and the recording sites of all neurons were reconstructed using the histology, the corresponding X-rays, and the X-rays on other tracks, as described in Feigenbaum \& Rolls (1991). The recording sites are shown in Fig. 7.

\section{RESULTS}

Recordings were made from 154 neurons in 39 daily recording sessions during the performance of the delayed match to sample task. The results are described in terms of four different experiments. The experiments were run in successive periods occupying up to several weeks each, given that tracks were not run at weekends.

\section{Experiment 1:}

Of 50 neurons analyzed for stimulus selectivity, 6 responded selectively to some of the presented images with an increase in firing rate more than to other images (evaluated by a KruskalWallis nonparametric test $(\mathrm{p}<0.001)$ with a MannWhitney post-hoc test, $\left.{ }^{*}=p<0.05, * *=p<0.01\right)$. All six neurons showed a smaller response to a stimulus the second time it was shown within a trial when compared with the first (see Fig. 2). Further, this response was reduced even when up to two other intervening non-rewarded nonmatching images were shown between the first (sample) and second (match) presentations of an image on a trial. (The response to the second presentation was: 0 images inserted $=38 \%+/-7 \%$ of the firing rate to the first presentation, $\mathrm{p}<0.01 ; 1$ image intervening $=49 \%+/-24 \%, \mathrm{p}<0.05 ; 2$ images intervening $51 \%+/-21 \%, \mathrm{p}<0.05$. $\mathrm{P}$ values refer to the difference between first (sample) and second (match) firing rates).

It was also found that the firing response of these 6 neurons 'reset' after each trial, and that the response to the first (sample) presentation of an image on a trial was always large (for an effective 

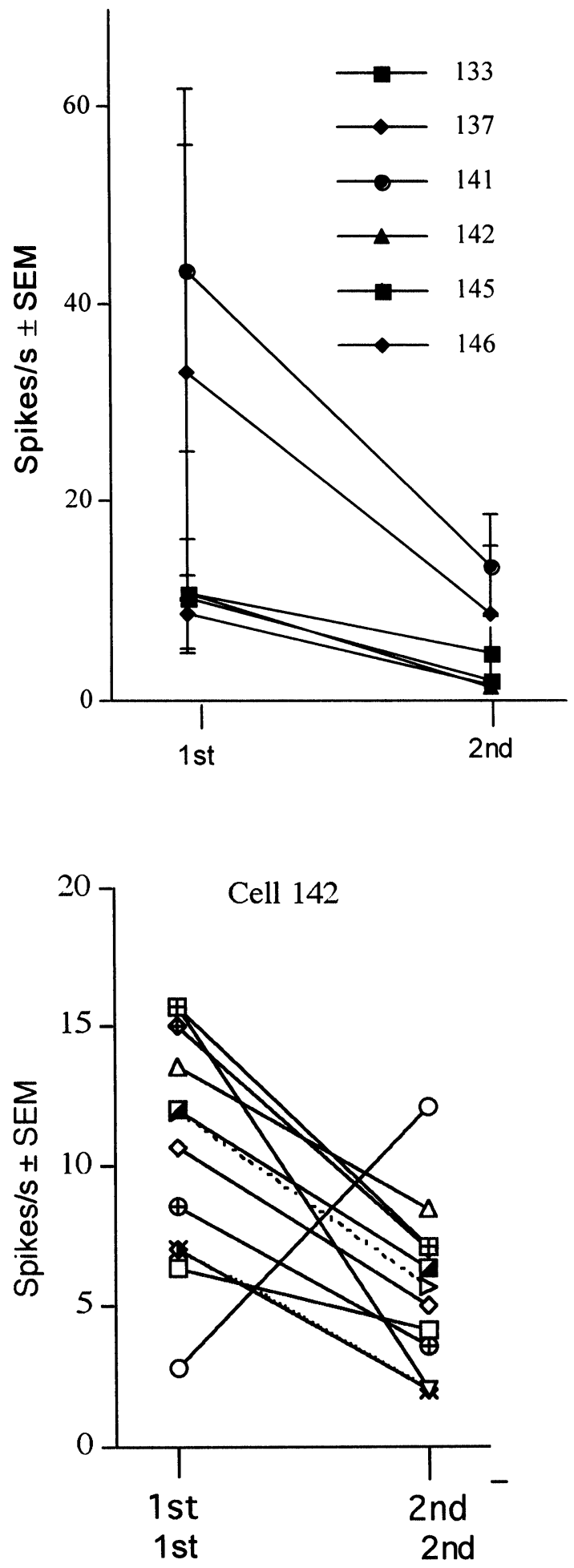

\section{Experiment 1:}

Fig. 2a: Responses of six cells to repeated presentations of one image within trials. All cells responded less to the second (match) presentation within a trial than to the same stimulus when shown as a sample $(\mathrm{P}<0.01$, Mann-Whitney test).

Fig 2b: (bottom) An example of the firing rates of a single cell responding to the first (sample) and the second (match) presentation of an image ( $p<0.05$, Mann-Whitney test). The resetting process is rather robust and was observed in 10 out of 11 trials. 
stimulus), even when that image had been shown very recently on the preceding trial as a match image to which the response was reduced (Fig. 3a). This comparison was possible in the experiments

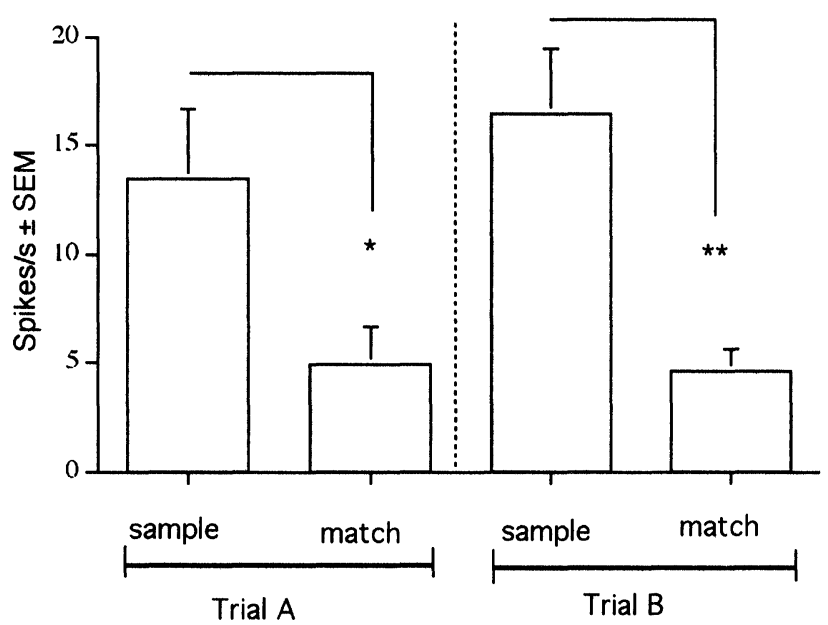

Fig. 3: b. (bottom) Example of one neuron's response to the presentation of one image within and between trials of the delayed match to sample task. The timing of the onset of stimuli within and between trials is shown. The inter-trial interval is shorter than the interval between sample and match presentation in this trial. we designed because the delay of presentation of an image within trials could be longer than the delay of presentations of images between trials (Fig 3b).

\section{Experiment 1: 'Resetting' between trials}

Fig. 3: a: (top) 'Resetting' of neuronal responses between trials. Neuronal responses are shown to familiar images that were repeatedly presented within one trial (as the sample and match), and were then shown again on the immediately succeeding trial. See text for interpreta-tion. $\mathrm{p}<0.001$ in a Kruskal -Wallis test; ${ }^{*}=$ $\mathrm{p}<0.01$ and $* *=\mathrm{p}<0.005$ in a Wilcoxon post-hoc test. The mean and SEM of the responses of the six neurons on one trial and on an immediate succeeding trial are shown.
TRIAL 1

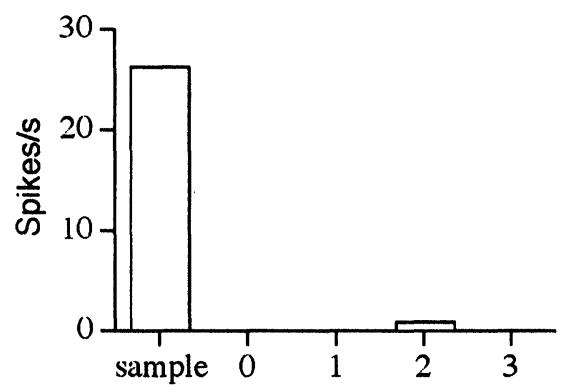

TRIAL 2

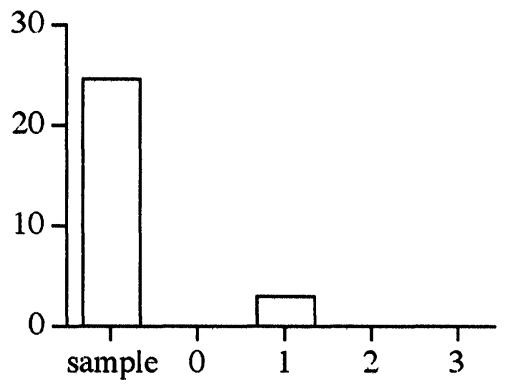

$4 \mathrm{~s}$

$3.2 \mathrm{~s}$ 
No difference was found in the firing rate response to an image that a cell responded to when rewarded (when shown as a match image) and to the same image when not rewarded (when shown as a non-match image) in another trial (response to match image normalized to $100 \%$, non-match image $107 \% \pm 11$ SEM). That is, the main effect observed for these six neurons was that the response to the sample was larger than to a match or a non-match image provided that it was an image to which the neuron was tuned. The remaining 46 cells in this first experiment that were not stimulus selective were not further analyzed.

\section{Experiments 2-4}

In Experiments 2-4, a new set of images, which included images of the same object viewed from different angles, was introduced in each experiment for use with the delayed match-tosample task. These cells were driven by visual stimuli, but most cells were not stimulus selective, and the analysis focused on the difference between the response of the neurons to the sample and to the later images within a trial.

Consistent with what we found in Experiment 1, of the 40 analyzed neurons, 34 responded more to an image when it was the sample than when it was the match on a given trial, and in addition 'reset' their responses between trials so that they responded more to the sample than to the match presentation of an image even when the image had been shown very recently as the match image on the preceding trial. Whereas firing rates were reduced when presenting an image twice within a trial, this reduction disappeared by the start of the next trial (see Fig. $4 ; p<0.001$ in a Kruskal-Wallis test; $*=p<0.01$ and $* *=p<0.005$ in a Wilcoxon post-hoc test).
When analyzing the effect of intervening images between sample and match image on the modulation of neuronal activity, it was found that up to two images could be inserted between the sample and match images without affecting the modulatory response (see Fig. 5; p $<0.001$ in a Kruskal-Wallis test; ${ }^{*}=\mathrm{p}<0.01$ and ${ }^{* *}=\mathrm{p}<0.005$ in a Wilcoxon post-hoc test).

To test whether the modulation of firing activity was simply due to the images being shown as a sample (first image shown in the task) and thereby only coding for the first position of images in the task (which would not require any memory process), the firing rates of cells during the presentation of familiar images that were shown in the second position (the first match image) were compared with the firing rates of these cells when another familiar image was shown later within the trial. The sample image was always a novel image in these trials. Under these conditions, cells still reduced their firing rate when a familiar image was shown again within a trial. Figure 6 shows the results of 10 cells given 16 trials each. The statistical significance of the firing reduction for each cell ranged between $p<0.01$ to $p<0.0001$.

Throughout Experiments 2 to 4, no difference was seen in the firing rate responses to familiar images that were rewarded (match image) and to familiar images that were not rewarded (non-match image) on other trials. The response to the nonmatch images was $94 \% \pm 13$ SEM of the response to the match images. Thus neither the match vs. non-match nor the reward vs. non-reward status of an image influenced the response of perirhinal cortex neurons to that image. In addition, sample images produced a larger response than non-match responses $\left(\mathrm{P}<0.001\right.$ in a Kruskal-Wallis test; ${ }^{*}=$ $\mathrm{p}<0.01$ in a Wilcoxon post-hoc test), thereby demonstrating that the lick response of the monkey was not responsible for the reduction in neuronal activity. 


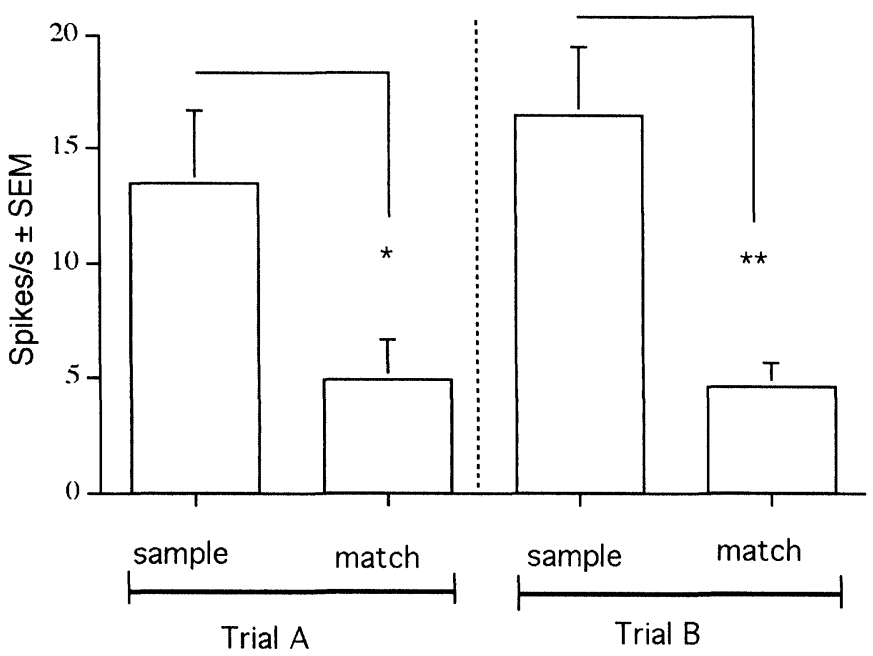

\section{Experiments 2-4: 'Resetting' of neuronal responses between trials.}

Fig. 4: Responses to familiar images that were used as sample and match stimuli on one trial ('A'), and then with only a short delay as the sample on the next trial ('B') is shown. A population of 34 out of 40 analyzed neurons were found to show resetting characteristics ( $p<0.001$ in a Kruskal -Wallis test; $*=p<0.01 * *=p<0.005$ in a Wilcoxon post-hoc test). The mean and SEM of the responses of the 34 neurons on one trial and on an immediately succeeding trial are shown.
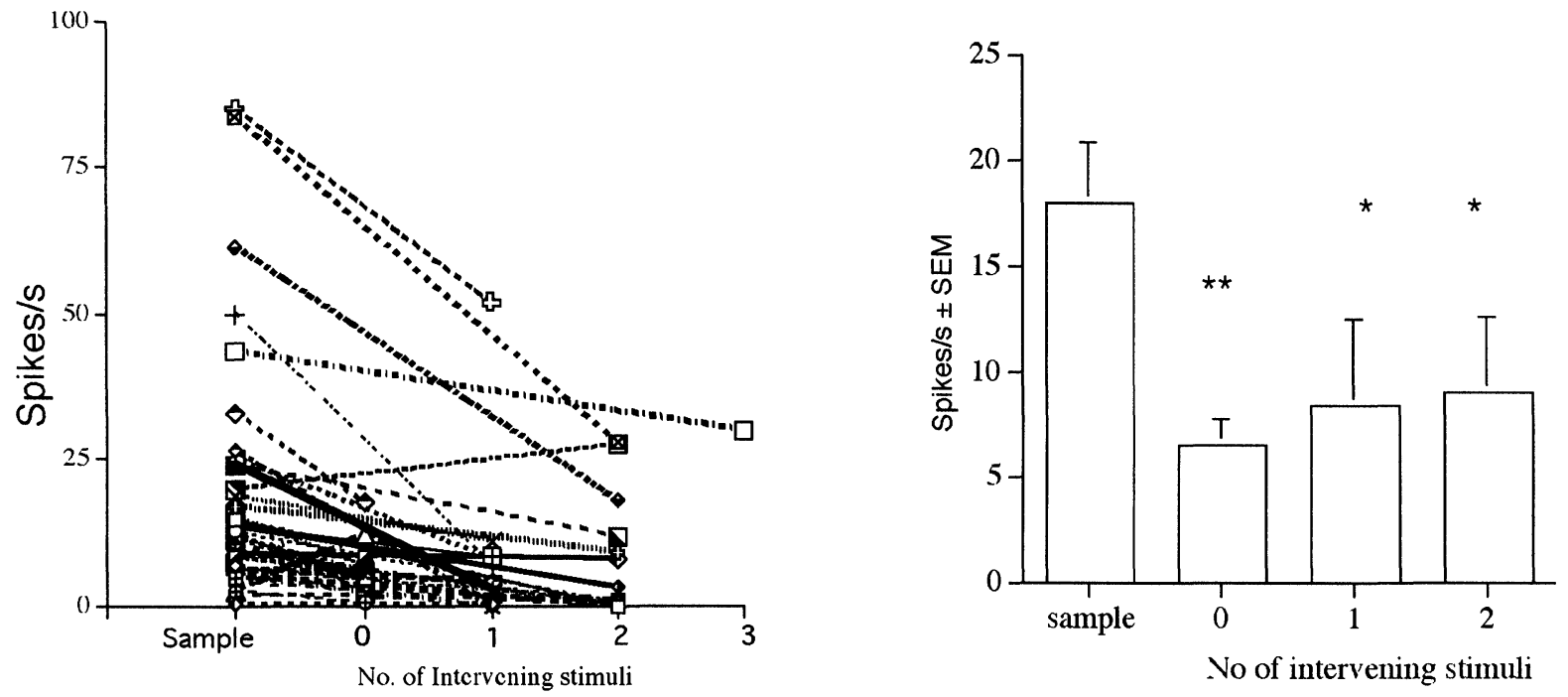

Fig. 5: All experiments: Effect of intervening stimuli on the modulation of neuronal responses. Left: Samples of single neuronal responses during sample and match presentation with 0-3 images intervening in between presentations. Right: statistical analysis of 44 neuronal responses showed a significant difference for up to two images $(\mathrm{P}<0.001$ in a Kruskal-Wallis test; $*=\mathrm{p}<0.01$ and $* *=\mathrm{p}<0.005$ in a Wilcoxon post-hoc test $)$. 


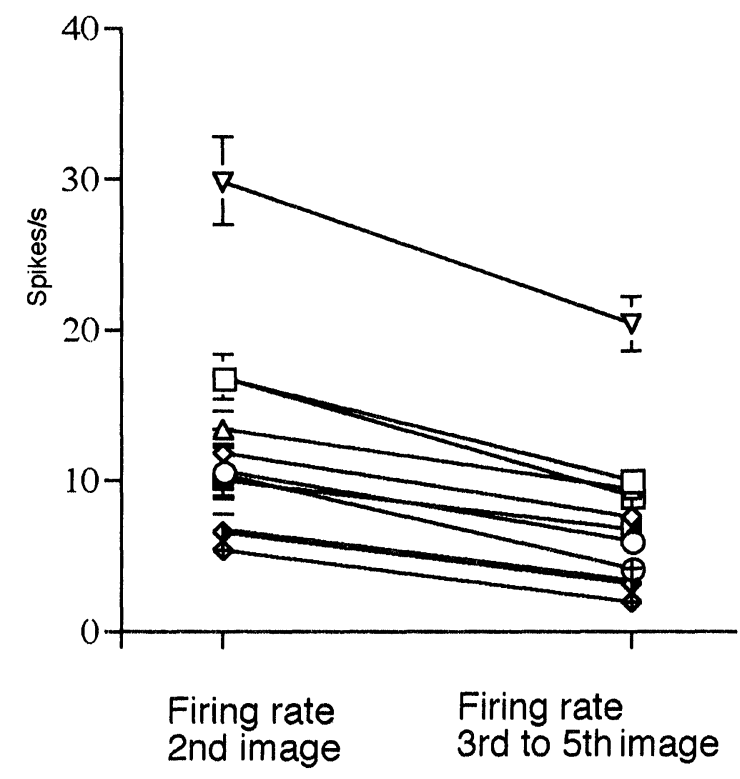

\section{Experiments 2-4: Modulation of firing for nonsample images.}

Fig. 6: The modulation of neuronal responses by stimulus repetition is not limited to images shown as samples. Shown in this figure are the modulation of responses of cells by familiar images within trials when a novel image is shown as a sample and a familiar image is shown as a first match image on position two $\left(2^{\text {nd }}\right.$ image shown) and again on a later position $\left(3^{\text {rd }}\right.$ to $\left.5^{\text {th }}\right)$. Neurons modulated their response to the second familiar image shown. The responses of 10 cells in 16 trials each are shown. Differences for firing rates $2^{\text {nd }}$ compared with 3 $5^{\text {th }}$ presentation ranged between $\mathrm{p}<0.01$ to $\mathrm{p}<0.0001$.

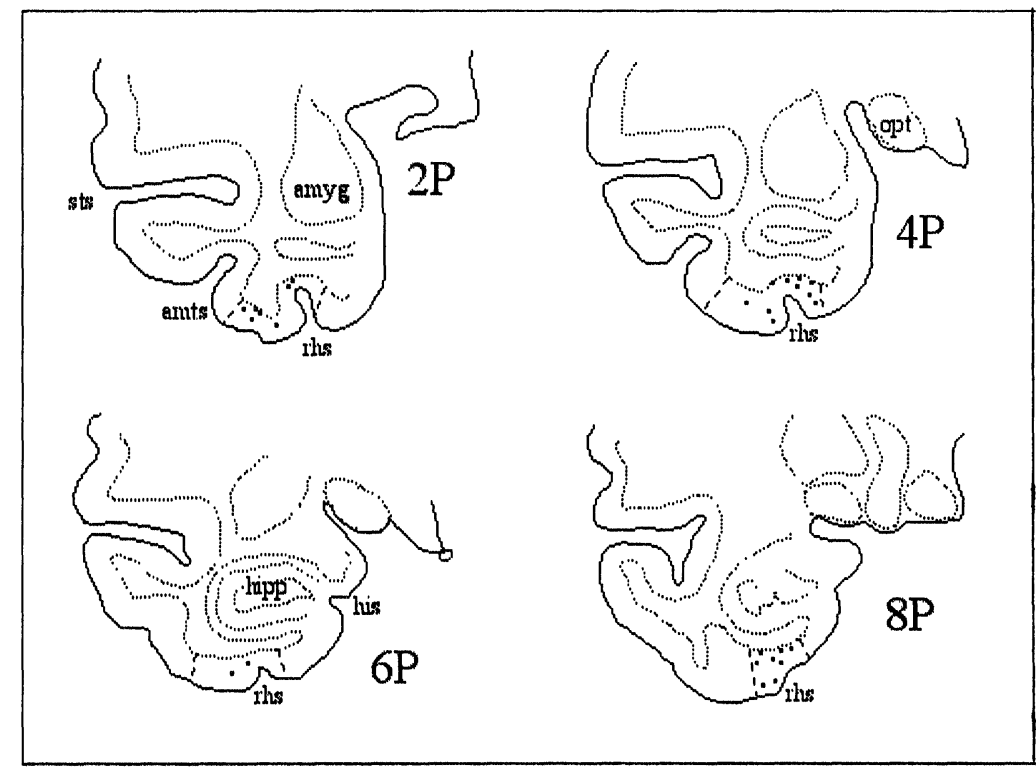

Fig. 7: Anatomical reconstruction of the sites in the perirhinal cortex at which the neurons were recorded, shown on coronal sections. Hatched lines show the boundaries of the perirhinal cortex. Dots designate the recording locations within electrode tracks that were found in the histological analysis. All co-ordinates refer to $\mathrm{mm}$ posterior to (P) the sphenoid (see Feigenbaum \& Rolls, 1991). 


\section{DISCUSSION}

The novel observation in this study was that neuronal responses in the macaque perirhinal cortex were not only modulated on a short-term basis with a trial, with a greater response to a sample than to a match image, but could be reset between trials. As shown in Experiments 1 (Fig. 2a, 2b) and Experiments 2 to 4 (Fig. 4), neuronal responses to images presented a second time within a trial (as a match stimulus) were greatly reduced. When the trial was over, however, the neuronal responses appeared to be 'reset', so that the same image presented as a sample on the next trial elicited a large response. It was found that this occurred even when the delay between the presentations of a particular image was much less between trials than within a trial. This resetting mechanism appears to be independent of the position of the image within the trial. When a familiar image is shown the first time on position two (as a match image), firing rates are high. When a second familiar image is shown later in the trial, firing rates are reduced. In the next trial, firing rates are high again. This shows that the firing rate modulating mechanism is not simply a 'sample image detector' that only indicates the first image position in a trial, but that the type of image (familiar vs. novel) is coded in the modulation.

Some other studies have described neurons that responded particularly to images that were shown very recently to the monkey ('recency neurons', see Brown and Xiang, 1998; Xiang and Brown, 1998). Taken together, the evidence presented here and in the other studies suggests that the perirhinal cortex (in conjunction with area TE, see Baylis and Rolls, 1987; Buffalo et al., 1999; Rolls and Deco, 2002) plays a pronounced role in the short term memory for visual information. The investigation described here provides evidence that the perirhinal cortex is actively involved in this short term memory process, rather than passively reflecting the recency with which a particular stimulus has been seen.

To consider this point more fully, the observations made in the present study show that the response changes of perirhinal cortex neurons found in short term memory tasks are not just simple decay-related effects. As shown in Fig. 3b, the delay within a task between showing an image the first (sample) and the second (match) time within a trial can be longer than the delay between showing the image as a match image on one trial and showing the same image again as the sample on the next trial. In this situation, the neurons can respond more to the image when it is being shown as a sample in the match-to-sample task, and the neuronal responses cannot be accounted for simply by a time-related decay function. There appears to be active resetting between trials of the short term memory task. On this evidence (see Figs. 2 to 4), one has to assume that the resetting is dependent on the beginning and ending of the trial, and therefore has working-memory qualities.

Further support for the proposal that the perirhinal cortex plays a role of in working memory is provided by the work of Miller et al. (1991a; $1991 \mathrm{~b}$; 1993) who showed that the responsiveness of neurons decreases with repeated stimulus presentation at interstimulus intervals of $2 \mathrm{~s}$ but not at intervals longer than $6 \mathrm{~s}$. The neuronal responsiveness recovered after a 5-min period of no stimulus presentation. In addition to this shortterm memory process, they also found an active enhancement of neuronal response that followed working memory-like dynamics. In addition, resetting of neuronal response modulation in a working memory -like fashion has been observed in the IT by others (see Suzuki, 1999, for a review). Further evidence for a role of the perirhinal cortex in short-term visual memory storage was also found in a lesion study. Rhesus monkeys with perirhinal cortex lesions were 
impaired in a delayed matching to sample task if a delay of up to $4 \mathrm{~s}$ was used. No impairment was found at longer delays (Hampton \& Murray, 2000). Because there are anatomical connections between the perirhinal cortex and the prefrontal cortex (Suzuki \& Amaral, 1994), and because the prefrontal cortex is involved in the direction of attention within tasks and working memory (Dias \& Aggleton, 2000; Duncan \& Owen, 2000; Rolls $\&$ Deco, 2002), one could postulate that the importance of the task-related prefrontal input to the perirhinal cortex is to direct attention towards memorizing images that are task-relevant. Evidence for such a theory comes from further studies that have shown that in sequential delayed match-to-sample trials, some neurons in the perirhinal cortex of primates respond to the position of images in such sequential trials, more than they do to the identity of individual images. Some neurons responded selectively to a visual cue that indicated that this was the first part of a trial (independently of which image was shown in that part of the trial), other neurons responded to the visual cue that indicated that the last part of a trial, and yet others responded to a visual cue that indicated an intermediate part of a trial (Liu \& Richmond, 2000). Additional studies also emphasized that attention plays an important role in the modulation of neuronal responses in perirhinal cortex (Chelazzi et al., 1998; Liu \& Richmond, 2000).

The results of these studies demonstrate that the perirhinal cortex is not just involved in the visual analysis or discrimination of images but also involved in the analysis of visual events according to their role in a task. The evidence thus suggests that activity in the perirhinal cortex is directly modulated by cortical areas that direct and modulate levels of attention and keep track of the complex temporal and logical schedules that are involved in tasks such as delayed match-to-sample tasks. The origin of this modulatory influence could be the prefrontal cortex (Rolls \& Deco, 2002).

It was of interest that neither the match vs. non-match nor the reward vs. non-reward status of an image influenced the response of perirhinal cortex neurons to that image. The main effect found in this study was that if an image was being shown the first time as a stimulus in the working memory task, then the response to the image was large. This result may reflect some active setting up for that particular image to be held in working memory as the to-be-remembered stimulus for that trial (see Rolls \& Deco, 2002). Recognition of this image later on as indicated by the reduced firing rate could be used by an analysis system to solve the task. The system would require more information than simply the recognition of an image shown previously within the task. We postulate that the prefrontal cortex, inferotemporal cortex, and limbic structures that code the reinforcer are also required by the brain to solve the working memory task. In conclusion, the findings described in this paper support the hypothesis that the perirhinal cortex plays an active role in visual working memory processes. The images appear to be loaded into working memory that is deleted after each trial.

\section{ACKNOWLEDGEMENT}

This research was supported by the Medical Research Council Interdisciplinary Research Centre for Cognitive Neuroscience.

\section{REFERENCES}

Baylis G, Rolls E. 1987. Responses of neurons in the inferior temporal cortex in short term and serial recognition memory tasks. Exp Brain Res 65: 614-622. 
Booth M, Rolls E. 1998. View-invariant representations of familiar objects by neurons in the inferior temporal visual cortex. Cerebral Cortex 8: 510-523.

Brown M, Xiang J. 1998. Recognition memory: neuronal substrates of the judgment of prior occurrence. Prog Neurobiol 55: 149-189.

Buckley MJ, Gaffan D, Murray EA. 1997. Functional double dissociation between two inferior temporal cortical areas: perirhinal cortex versus middle temporal gyrus. J Neurophysiol 77: 587-598.

Buffalo EA, Ramus SJ, Clark RE, Teng E, Squire LR, Zola SM. 1999. Dissociation between the effects of damage to perirhinal cortex and area TE. Learn Mem 6: 572-599.

Chelazzi L, Duncan J, Miller E, Desimone R. 1998. Responses of neurons in inferior temporal cortex during memory guided visual search. J Neurophysiol 80: 2918-2940.

Dias R, Aggleton JP. 2000. Effects of selective excitotoxic prefrontal lesions on acquisition of nonmatching- and matching-to-place in the T-maze in the rat: differential involvement of the prelimbicinfralimbic and anterior cingulate cortices in providing behavioural flexibility. Eur J Neurosci 12: 4457-4466.

Duncan J, Owen A. 2000. Common regions of the human frontal lobe recruited by diverse cognitive demands. Trends Neurosci 23: 475-483.

Feigenbaum JD, Rolls ET. 1991. Allocentric and egocentric spatial information processing in the hippocampal formation of the behaving primate. Psychobiology 19: 21-40.

Hampton R, Murray E. 2000. Deficits following perirhinal cortex lesions: representation or retention? Society of Neuroscience Meeting, New Orleans: (abstract) 205.2.

Liu Z, Richmond B. 2000. Response differences in monkey TE and perirhinal cortex: stimulus association related to reward schedules. J Neurophysiol
83: 1677-1692.

Miller E, Gochin P, Gross C. 1991a. Habituation-like decrease in the responses of neurons in inferior temporal cortex of the macaque. Vis Neurosci 7: 357-362.

Miller E, Li L, Desimone R. 1991b. A neural mechanism for working and recognition memory in inferior temporal cortex. Science 254: 1377-1379.

Miller E, Li L, Desimone R. 1993. Activity of neurons in anterior inferior temporal cortex during a shortterm memory task. J Neurosci 13: 1460-1478.

Miyashita Y, Kameyama M, Hasegawa I, Fukushima T. 1998. Consolidation of visual associative longterm memory in the temporal cortex of primates. Neurobiol Learn Mem 1: 197-211.

Miyashita Y, Okuno H, Tokuyama W, Ihara T, Nakajima K. 1996. Feedback signal from medial temporal lobe mediates visual associative mnemonic codes of inferotemporal neurons. Brain Res Cogn Brain Res 5: 81-86.

Rolls E.T. 2000. Functions of the primate temporal lobe cortical visual areas in invariant visual object and face recognition. Neuron 27: 205-218.

Rolls E, Deco G. 2002. Computational Neuroscience of Vision. Oxford, UK: Oxford University Press.

Suzuki W, Amaral D. 1994. Perirhinal and parahippocampal cortices of the macaque monkey: cortical afferents. J Comp Neurol 350: 497-533.

Suzuki W, Amaral D. 1994. Topographic organization of the reciprocal connections between the monkey entorhinal cortex and the perirhinal and parahippocampal cortices. J Neurosci 14: 1856-1877.

Suzuki W 1999. The long and the short of it: memory signals in the medial temporal lobe. Neuron 24 : 295-298.

Xiang J., Brown M. 1998. Differential neuronal encoding of novelty, familiarity and recency in regions of the anterior temporal lobe. Neuropharmacology 37: 657-676. 

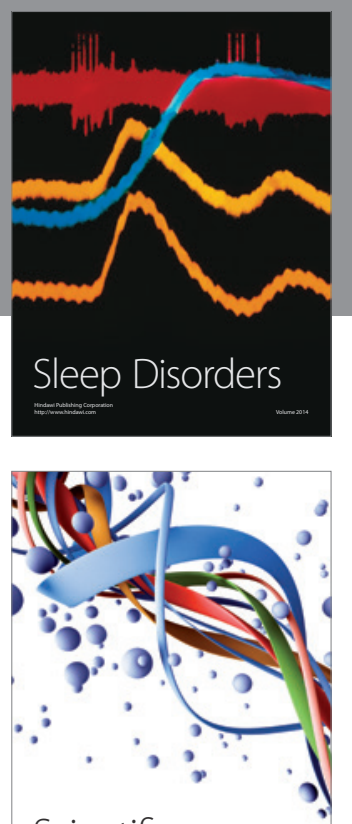

Scientifica
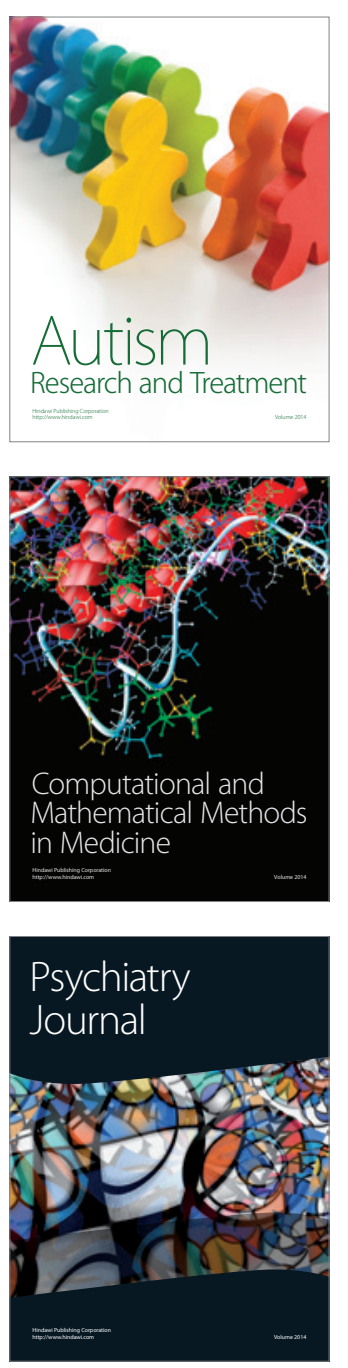
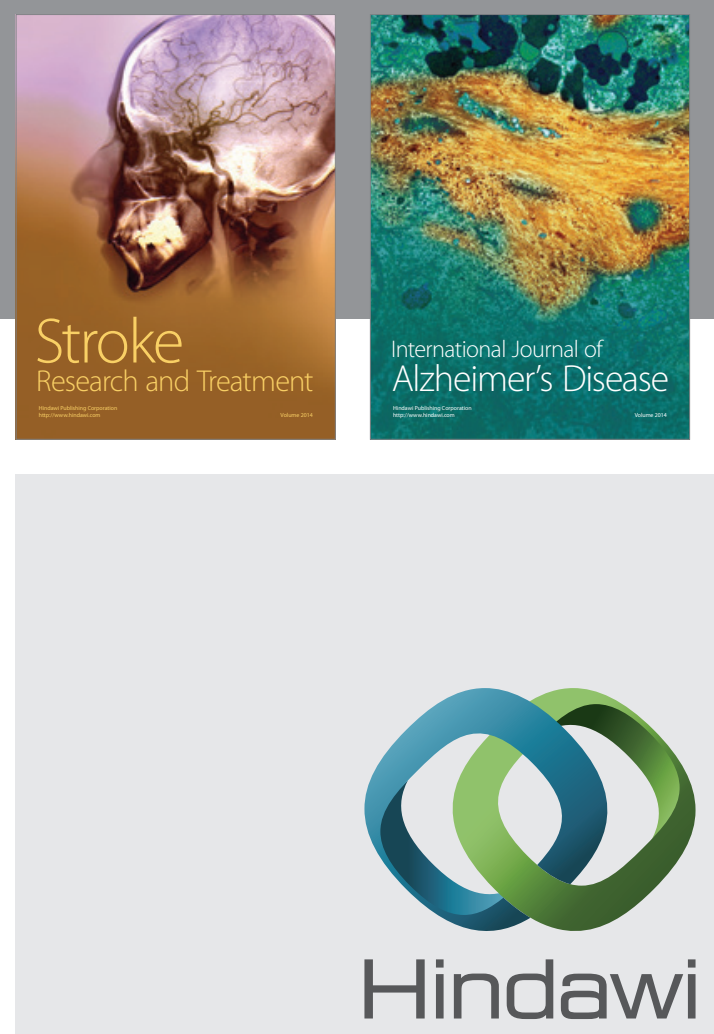

Submit your manuscripts at

http://www.hindawi.com
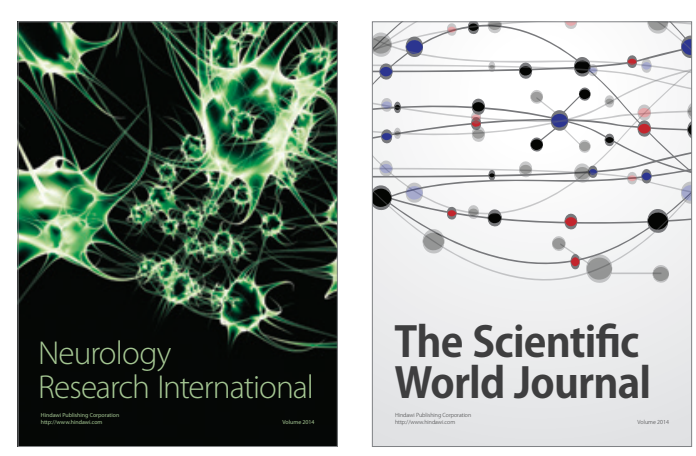

The Scientific World Journal

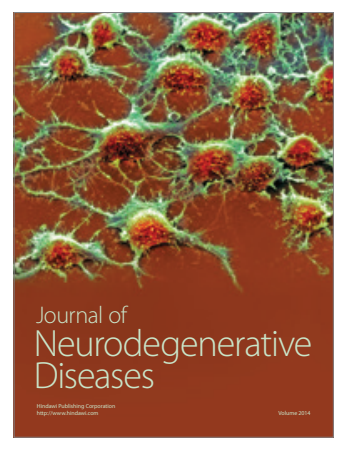

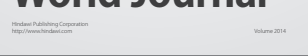

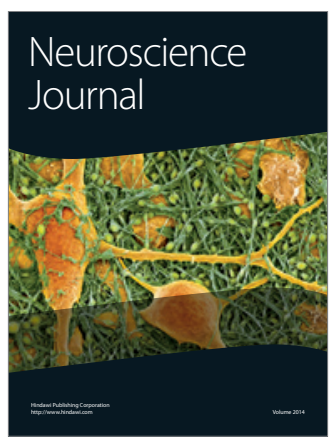

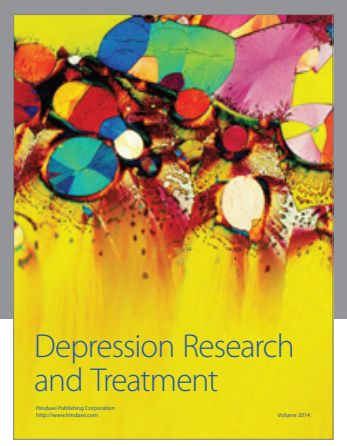
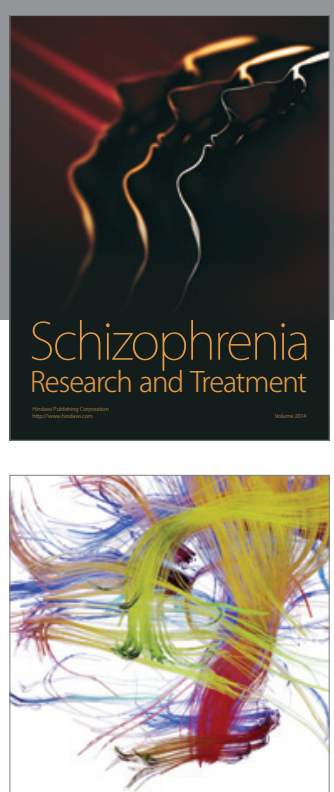

Brain Science

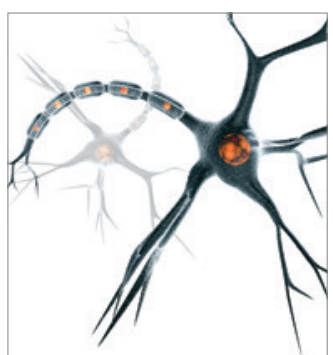

Neural Plasticity
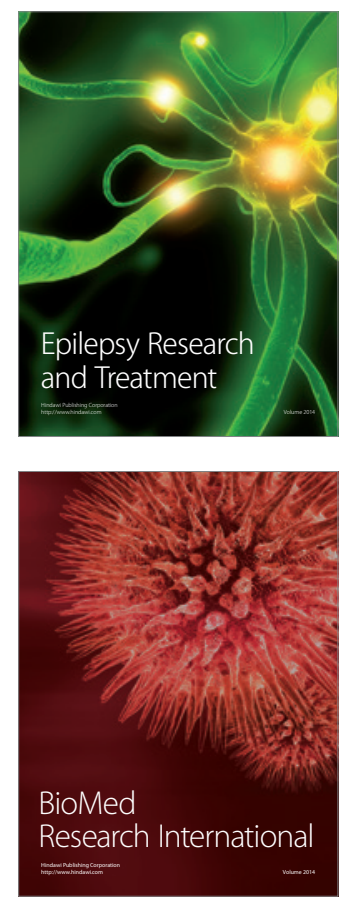

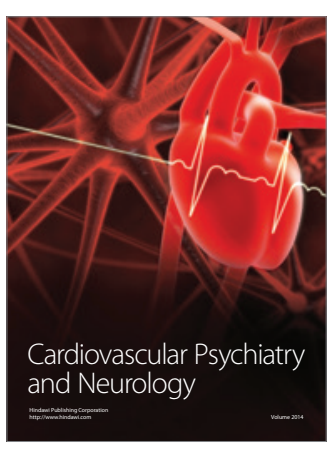

Parkinson's

Disease
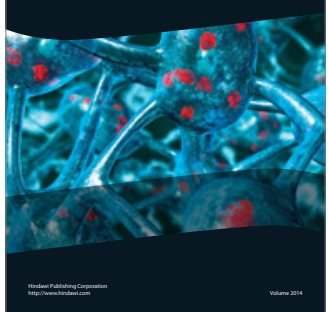